\title{
Propiedades psicométricas del Test de Dependencia al Teléfono Móvil (TDMB) en Argentina y sus relaciones con la impulsividad
}

\author{
Psychometric Properties of the Mobile Phone Dependency Test \\ (TDMB) in Argentina and its Relationship with Impulsivity
}

\author{
Marian Durao ${ }^{1}$ \\ http://orcid.org/0000-0002-1860-4780 \\ (iD) \\ Edgardo Etchezahar ${ }^{2}$ \\ http://orcid.org/0000-0002-3289-194X \\ (iD \\ Joaquín Ungaretti ${ }^{3}$ \\ https://orcid.org/0000-0003-1185-9139 \\ iD \\ Carolina Calligaro 4 \\ https://orcid.org/0000-0003-0924-8734 \\ iD \\ 1.Facultad de Psicología y Ciencias Sociales, Universidad de Flores, Argentina \\ ${ }^{2}$ CIIPME- CONICET, Universidad de Buenos Aires, Argentina \\ ${ }^{3}$ CONICET, Universidad de Buenos Aires, Argentina \\ ${ }^{4}$ Facultad de Humanidades y Ciencias Sociales, Universidad de Palermo, Argentina
}

\begin{abstract}
Resumen. El uso de teléfonos celulares ha aumentado exponencialmente en la mayoría de los países del mundo. Desde una perspectiva psicológica, se han desarrollado diferentes técnicas para evaluar el nivel de dependencia hacia el uso del teléfono móvil, y el test TDMB es una de las más utilizadas. Objetivo. El objetivo principal del trabajo fue realizar la adaptación y validación al contexto argentino del cuestionario TDMB y sus relaciones con la impulsividad. Método. Participaron del estudio 339 adultos residentes en Argentina, con edades entre los 18 y 65 años $(M=37.66$; DT $=12.87)$, siendo el $74.3 \%$ mujeres. La batería de evaluación incluyó el TDMD, la UPPS-P para evaluar impulsividad y variables ad-hoc relacionadas con el uso del teléfono celular. Resultados. Se obtuvieron propiedades psicométricas adecuadas para la versión argentina del TDMB. Asimismo, se observaron relaciones entre todas las dimensiones del cuestionario y la impulsividad, como también diferencias según la edad y el sexo de los participantes. Se discuten los alcances y límites de la prueba, así como sus relaciones con la impulsividad y el uso cotidiano del teléfono móvil.
\end{abstract}

Palabras clave. Teléfono móvil, dependencia, test, impulsividad, abstinencia.

Abstract. The use of cell phones has increased exponentially in most countries of the world. From a psychological perspective, different techniques have been developed to assess the level of dependence towards the use of the mobile phone, the TDMB test being one of the most used. Objective. The aim of the research was to adapt and validate the TDMB and its relations with impulsivity. Method. 339 adults residing in Argentina participated in the study, with ages between 18 and 65 years $(M=37.66$; SD $=12.87$ ), of which $74.3 \%$ were women. The evaluation included the TDMD, the UPPS-P to evaluate impulsivity and ad-hoc variables related to cell phone use. Results. Adequate psychometric properties were observed for the Argentine version of the TDMB. Likewise, relationships were observed between all dimensions of the questionnaire and impulsivity, as well as differences according to the age and gender of the participants. The scope and limits of the test are discussed, as well as its relationships with the impulsivity and daily use of the mobile phone.

Keywords. Mobile phone, Dependence, Test, Impulsivity, Abstinence.

${ }^{1}$ Marian Durao. Universidad de Flores, Facultad de Psicología y Ciencias Sociales. Dirección postal: Av. Rivadavia 5741, Caballito, CABA, Argentina. E-mail: marian.durao@gmail.com

²Edgardo Etchezahar. CIIPME-CONICET, Universidad de Buenos Aires, Universidad Nacional de Lomas de Zamora, Argentina. E-mail: edgardoetchezahar@ psi.uba.ar

${ }^{3} J o a q u i ́ n$ Ungaretti. CONICET, Universidad de Buenos Aires, Universidad Nacional de Lomas de Zamora, Argentina. E-mail: ludgleyjungaretti@psi.uba.ar ${ }^{4}$ Carolina Calligaro. Facultad de Psicología y Ciencias Sociales, Universidad de Flores, Argentina. E-mail: carocall@yahoo.com 


\section{Introducción}

El uso de teléfonos celulares se ha incrementado de manera exponencial en la mayoría de los países del mundo, a tal punto, que hay tantas personas como dispositivos móviles (Chóliz et al., 2016). Se han señalado casos interesantes como el de Irlanda, donde hay más teléfonos móviles que personas (Chóliz et al., 2016). Por su parte, en Argentina estudios de IBOPE Media afirman que el índice de penetración -cantidad de teléfonos celulares por habitante- ha aumentado en los últimos dos años, lanzando la cifra de 39.9 millones de usuarios sobre 44.5 millones de habitantes en el país (Instituto Nacional de Estadísticas y Censos, 2017). En este sentido, el uso de telefonía celular con acceso a internet, ha modificado la cotidianeidad de gran parte de la población mundial, ocasionando, incluso, la emergencia de los problemas relacionados con la adicción al uso de los teléfonos móviles. Este fenómeno se ha convertido en una causa de preocupación para la salud mental en todo el mundo (Miller, 2012).

En el marco de la psicología, se sabe que la adicción o dependencia no es un problema causado solamente por el consumo de sustancias como drogas o alcohol, sino que, tal y como afirman Chóliz et al. (2017), ciertos patrones de conducta pueden causar problemas de dependencia de la misma forma que lo hacen algunas sustancias. Un ejemplo de esto es el juego patológico, como ha reconocido la Sociedad Americana de Psiquiatría (APA, 2018), pero es especialmente relevante también en el caso de las Ilamadas adicciones o dependencias comportamentales (Grant et al., 2010) o en ausencia de sustancias (Echeburúa, 1999). En este sentido, Chóliz et al. (2017), sostienen que una de las formas más recientes, y de características sumamente específicas, de dependencias comportamentales son aquellas generadas por el uso disfuncional de las Nuevas Tecnologías de la Información y la Comunicación (en adelante TIC). La mayoría de las dependencias a la tecnología involucran el uso de Internet (Tsai \& Lin, 2003), de los teléfonos móviles (Chóliz, 2010) y de videojuegos (Griffiths et al., 2012).

En cuanto a la dependencia al teléfono móvil con acceso a internet, hay numerosos estudios científicos y evidencia clínica sobre los problemas psicológicos de carácter adictivo derivados del uso disfuncional de las redes sociales o la mensajería instantánea a través de este dispositivo (Chóliz et al., 2016; Griffiths, 2000). En esta línea, los síntomas más observados de dependencia al teléfono móvil son: uso excesivo; alto costo para la economía personal en relación con la cantidad de llamadas o la cantidad de mensajes enviados; problemas interpersonales por el uso desmedido; interferencia en las actividades académicas o laborales; tolerancia - un incremento gradual en la necesidad de uso del teléfono móvil a fin de conseguir un incremento en los niveles de satisfacción-, entre otros (Chóliz, 2010).

Continuando con el análisis de la sintomatología, Chóliz et al. (2017) sostienen que, tal como ocurre en el caso del juego patológico y otras dependencias, la adicción a los dispositivos tecnológicos se caracteriza por: a) necesidad cada vez mayor de hacer uso de la tecnología, a fin de obtener el mismo bienestar que en un principio -tolerancia-; b) aparición de reacciones emocionales negativas cuando no se puede utilizar la tecnología o cuando se ha pasado un largo tiempo sin poder usarla -síntomas de abstinencia-; c) el uso excesivo de la tecnología interfiere con actividades importantes de la vida cotidiana, ya sea del área profesional, familiar, o situaciones sociales; y d) presencia de serias dificultades al momento de tener que dejar de usar la tecnología, más allá de saber que este patrón de conducta es inapropiado o potencialmente peligroso.

Actualidades en Psicología, 35(130), 2021, 1-18 
Aunque son abundantes los criterios definitorios de adicción al teléfono móvil, existiría, sin embargo, una importante coincidencia y cohesión expresada en la dependencia con abstinencia, la tolerancia y la falta de control, que conduce a interferencias y abandono de las actividades cotidianas (Chóliz, 2010; Anestis et al., 2007; Verdejo-García et al., 2007).

Los procesos psicológicos que promueven la dependencia son el núcleo de la adicción a los dispositivos tecnológicos, entre los cuales la impulsividad podría tener un papel esencial, sobre todo desde su íntima relación con la búsqueda de sensaciones (De Sola Gutierrez et al., 2013). Tal y como ha sido propuesto por Billieux (2012), la impulsividad es un constructo multifactorial con un papel central en la psicopatología y, especialmente, en las adicciones.

Con la finalidad de evaluar empíricamente este constructo, se ha desarrollado la escala breve de comportamiento impulsivo UPPS-P1 (Cándido et al., 2012), la cual evalúa cinco rasgos relacionados con el comportamiento impulsivo: urgencia positiva, urgencia negativa, falta de premeditación, falta de perseverancia y búsqueda de sensaciones. La urgencia positiva hace referencia a la proclividad a sucumbir a impulsos fuertes bajo la influencia de emociones positivas; la urgencia negativa se entiende como la tendencia a actuar precipitadamente en presencia de estados afectivos negativos; la falta de premeditación es la tendencia a actuar sin tener en cuenta las consecuencias de los actos; la falta de perseverancia se entiende como la incapacidad para mantenerse inmerso en tareas que pueden ser aburridas, extensas o difíciles; por último, la búsqueda de sensaciones puede definirse como la propensión a participar en actividades nuevas, excitantes y/o peligrosas (Anestis et al., 2007; Verdejo-García et al., 2007).

En este sentido, Gutierrez (2014) sostiene que el objetivo final de conseguir un refuerzo gratificante se plasma en el uso excesivo del celular o la conexión constante a redes sociales, donde la impulsividad sería un acto irresistible en el que se experimenta un deseo intenso de realizar estas acciones, aunque el sujeto sepa que a veces pueden ser desencadenantes de consecuencias negativas (Salvo \& Castro, 2013). Este tipo de comportamientos responderían al modelo del circuito del refuerzo de Koob y Volkow (2010), en un lugar de transición de la impulsividad a la compulsión, marcado por el cambio del refuerzo positivo al negativo.

Por su parte, si bien en el DSM-V la impulsividad se encuentra dentro de la categoría de trastornos disruptivos, del control de los impulsos y otras alteraciones conductuales (Botero-Franco et al., 2016), esta quinta versión presenta un cambio importante que reemplaza el término 'adicción' por los 'Trastornos de uso y abuso', añadiendo el 'craving' o deseo intenso de consumir. Sin embargo, hay nueva terminología que no figura en los manuales diagnósticos para dar cuenta de los fenómenos relacionados con el abuso o uso excesivo del celular (Taneja, 2014), y aún resulta difícil determinar si Internet y las Nuevas Tecnologías en general son capaces de suscitar comportamientos adictivos o son, por el contrario, generados por los contenidos (Ma, 2011).

Es necesario considerar que las redes sociales, los juegos y otras actividades que se pueden encontrar en Internet, tienen efectos reforzantes para los usuarios cuando navegan, los cuales podrían hacer que la actividad se prolongue, a modo de reforzadores que luego podrían constituir reforzadores intrínsecos; una especie de "disfrute percibido" que, a largo plazo, sostiene el comportamiento de uso de estas aplicaciones y que pueden generar hábitos que en algunos casos podrían considerarse dañinos (Turel \& Serenko, 2012). Las Ilamadas adicciones comportamentales se caracterizan por una necesidad 
imperiosa de involucrarse en conductas que conllevan consecuencias negativas; a su vez, implican cadenas comportamentales que hasta completarse generan urgencia y tensión y se activan mediante señales internas y externas que dificultan en funcionamiento cotidiano (Pedrero-Pérez et al., 2018).

Numerosos autores consideran el exceso de uso de Internet como un concepto relacionado, fundamentalmente, con la magnitud de la conducta en términos de tiempo y frecuencia (Carbonell \& Oberst, 2015; Matute, 2016; Starcevic, 2012; Turel \& Serenko, 2012; Valencia-Ortiz \& Cabero-Almenara, 2019). Al respecto, Matute (2016) sostiene que esto no es adicción, como tampoco lo es el abuso de Internet, ya que esta conducta podría estar relacionada también con el uso intensivo por estudios o trabajo.

Es así que ciertos autores (Becoña, 2016; Matute, 2016) proponen el uso problemático como un concepto que define más adecuadamente el caso, alejándolo del concepto de adicción, pero considerando algunos elementos importantes que se relacionan con los riesgos que genera su uso. Además, considera algunas características que no son normales en la conducta individual, como el aislamiento de la familia y los amigos; el aplazamiento de alguna necesidad básica, como son la alimentación y el sueño; la falta de control de la impulsividad, etc., que es denominado uso patológico de Internet (Turel \& Serenko, 2012). Por su parte, otros autores proponen la denominación de uso compulsivo (Guertler et al., 2014).

En lo que todos están de acuerdo es que los manuales de diagnóstico más utilizados, como el de Clasificación Estadística Internacional de Enfermedades y Problemas Relacionados con la Salud, de la Organización Mundial de la Salud (CIE, OMS, 2018) y el Manual Diagnóstico y Estadístico de los Trastornos Mentales (DSM, 1994, 2013) de la American Psychiatric Association (APA), no consideran la existencia de este tipo de adicciones, salvo en el caso específico del juego patológico, y hacen una apreciación genérica de las adicciones sin sustancias (Carbonell, 2014; Carbonell \& Oberst, 2015; Caro, 2017; Cía, 2013; Matute, 2016; Starcevic, 2012; Valencia-Ortiz \& Cabero-Almenara, 2019). En definitiva, las adicciones tecnológicas no se encuentran reconocidas en las principales clasificaciones de trastornos mentales y esto condiciona los instrumentos para la medición de su uso (Simó et al., 2017).

Los mencionados elementos de dependencia al móvil se pueden evaluar mediante instrumentos de evaluación como The Mobile Phone Problem Usage Scale (Bianchi \& Phillips, 2005), The Mobile Phone Dependence Questionnaire (MPDQ; Toda et al., 2006), The Problematic Mobile Phone Use Questionnaire (Billieux et al., 2008) o The Scale of Selfperception of Text-Message Dependency (Igarashi et al., 2008) y Test de Dependencia al Teléfono Móvil (TDM) de Chóliz (2010).

El TDM evalúa las principales características de la dependencia a este tipo de dispositivos tales como tolerancia, abstinencia, control de los impulsos, uso excesivo y problemas asociados. En cuanto a las propiedades psicométricas de dicho instrumento, en el estudio original (Chóliz, 2012), el autor creó una evaluación compuesta por 22 ítems con una consistencia interna para el instrumento en su totalidad de $\alpha=.94$. Luego de realizar un análisis factorial exploratorio, el autor arribó a una solución factorial compuesta por tres dimensiones que reflejan el constructo: Abstinencia, el cual explicó un $42.69 \%$ de la varianza total; Falta de control y problemas derivados del uso, que explicó un $10.38 \%$ de la varianza total; Tolerancia e interferencia con otras actividades, que explicó un 5.64\% del total de varianza. Pese a la identificación de diferentes factores para trabajar con la 
evaluación, no se informó la consistencia interna para cada uno de ellos. Por último, el autor encontró diferencias estadísticamente significativas según el sexo, tanto en la escala total como en cada una de las tres dimensiones, donde las mujeres obtuvieron puntajes significativamente mayores al obtenido por los hombres de la muestra, coincidiendo con otros autores (Villafuerte-Garzón \& Vera-Perea, 2019). Con respecto a la edad de los participantes, esta se correlacionó positivamente con la percepción de problemas, tendencia que también ha sido confirmada en el estudio de Labrador y Villadangos (2010).

Años más tarde, Chóliz et al. (2016) seleccionaron los ítems más representativos de la versión original del TDM y desarrollaron la versión reducida (en adelante TDM Brief) de la prueba. Esta versión quedó conformada por 12 ítems que obtuvieron una adecuada consistencia interna global $(\alpha=.88)$. Luego de realizar un análisis factorial exploratorio, los autores arribaron a una estructura compuesta por cuatro dimensiones correlacionadas, en lugar de las cinco originales, que en su conjunto explicaron el $66.68 \%$ de la varianza total. El primer factor fue denominado Abstinencia y explicó el $44.08 \%$ de la varianza total, además de obtener una adecuada consistencia interna $(\alpha=.81)$. Por su parte, el segundo factor fue denominado Abuso e interferencia con otras actividades y explicó el 9.61\% de la varianza total $(\alpha=.70)$. En tercer lugar se obtuvo un factor al que denominaron Tolerancia y explicó el $6.76 \%$ del total de varianza $(\alpha=.75)$. Por último, se obtuvo un factor al que denominaron Falta de control y explicó el 6.22\% del total de varianza que, si bien presentó una consistencia interna inferior, resultó aceptable para los fines del estudio $(\alpha=.64)$. Otros estudios, como el realizado por Gamero et al. (2016), también aportaron evidencias de validez y confiabilidad para el Test original de Dependencia al Móvil de Chóliz et al. (2010).

Por otra parte, Ruiz-Palmero et al. (2016) encontraron que el TDM Brief mostró correlaciones fuertes y estadísticamente significativas entre el tipo de dispositivo con el que se conectan los adolescentes a Internet, con la dependencia a los dispositivos móviles y con la problemática que aparece cuando el empleo aumenta a niveles excesivamente altos o que pueden causar trastornos. A su vez, el TDM Brief obtuvo una correlación positiva con el patrón de uso del teléfono móvil y con la percepción de dependencia a él. La consistencia interna de la escala fue adecuada para dicho estudio $(\alpha=.94)$ (Simó et al., 2017).

Teniendo en cuenta lo anteriormente expuesto y que no se han hallado estudios en el contexto argentino que analicen la dependencia al teléfono móvil y su relación con la impulsividad, el objetivo general del estudio fue realizar la adaptación y validación al contexto argentino del Test de Dependencia al Móvil versión reducida (TDMB) de Chóliz et al. (2016), derivado del TDM en su versión original (Chóliz, 2010, 2012), para luego analizar sus relaciones con la impulsividad y una serie de preguntas relacionadas con el uso del teléfono celular. Asimismo, se estudiaron las relaciones entre las dimensiones del TDMB con el sexo y la edad de los participantes.

\section{Método}

\section{Participantes}

Los participantes se seleccionaron por medio de un muestreo no probabilístico, incidental, compuesto por 339 adultos de nacionalidad argentina, con edades comprendidas entre los 18 y 65 años $(M=37.66$; $D T=12.87)$. El $25.7 \%$ eran hombres $(n=87)$ y el $74.3 \%$ mujeres $(n=252)$. 


\section{Instrumentos}

Se emplearon mediciones de autoinforme mediante una batería de instrumentos de evaluación, que se detalla a continuación.

Test de Dependencia al Teléfono Móvil Breve. Se realizará la adaptación y validación al contexto argentino del cuestionario TDM Brief (Chóliz et al., 2016), derivado del TDM en su versión original (Chóliz, 2010, 2012). El cuestionario está conformado por cuatro dimensiones, con tres ítems cada una, que se corresponden con las dimensiones de la versión original de la prueba: abstinencia $(\alpha=.81)$, abuso e interferencia con otras actividades $(\alpha=.70)$, tolerancia $(\alpha=.75)$, falta de control $(\alpha=.64)$. En el presente estudio se utilizó un formato de respuesta tipo Likert que va de 1 = totalmente en desacuerdo a 5 = totalmente de acuerdo.

Comportamiento impulsivo. Se administró la versión española de la escala breve de comportamiento impulsivo UPPS-P (Cándido et al., 2012), la cual consta de 20 ítems que evalúan cinco rasgos relacionados con el comportamiento impulsivo: urgencia positiva ( $\alpha$ $=.61)$, urgencia negativa $(\alpha=.68)$, falta de premeditación $(\alpha=.78)$, falta de perseverancia $(\alpha=.79)$ y búsqueda de sensaciones $(\alpha=.81)$. Los ítems se puntúan en una escala tipo Likert de cinco anclajes, que van de $1=$ no estoy en absoluto de acuerdo a $5=$ totalmente de acuerdo.

Preguntas relacionadas con el uso del celular. Se realizó una serie de preguntas ad hoc sobre el uso del celular. Por un lado, se indagó por la frecuencia con la que los individuos llevan a cabo ciertas acciones relacionadas al uso del celular (e.g. Del 0 al 10, ¿Cuán dependiente sos del celular?; Me han Ilamado la atención o me han hecho alguna advertencia por utilizar mucho el celular; Me he excedido con el uso del celular; Cuando me aburro, utilizo el celular).

Cuestionario de datos personales. Por último, se indagó por el sexo, la edad y la clase social autopercibida de los participantes.

\section{Procedimiento y análisis de datos}

Para la adaptación del TDM Brief al contexto argentino, se siguieron los estándares metodológicos internacionales recomendados por la International Test Commission (ITC) (Hambleton, 1994; Muñiz et al., 2013). Además, se siguieron los pasos realizados por Chóliz et al. (2016) para la selección de los ítems que conformaron la evaluación que luego sería administrada a una muestra piloto compuesta por 45 participantes.

En una segunda fase, a partir de las recomendaciones de los participantes, se reformularon una serie de ítems y descartaron otros hasta arribar a la versión final de la escala. Estos pasos permiten el ajuste idiomático del instrumento al contexto argentino y fueron útiles para identificar los ítems que mejor representaran los constructos evaluados.

Los participantes que formaron parte de esta investigación lo hicieron de manera voluntaria y anónima, luego de brindar su consentimiento. Previamente a la toma de datos, se informó a los participantes que la información revelada sería utilizada con fines exclusivamente académico-científicos, según lo indica la Ley Nacional 25.326 de protección de datos personales. Para llevar a cabo los análisis correspondientes, se utilizó el software SPSS para Windows versión 21.0 y el EQS 6.1 (AFC). 


\section{Resultados}

En primer lugar, se analizaron los ítems de la escala TDMB, los cuales conforman las cuatro dimensiones del constructo (Tabla 1).

En cuanto a las propiedades psicométricas, los análisis descriptivos que se observan en la Tabla 1 resultaron adecuados tanto en términos de la media y el desvío como de la

Tabla 1

Análisis descriptivo, correlación ítem-total y alfa si se elimina el elemento

\begin{tabular}{|c|c|c|c|c|c|c|}
\hline & M & DT & $S$ & K & rjx & $\alpha .-x$ \\
\hline \multicolumn{7}{|l|}{ Abstinencia $(\alpha=.85)$} \\
\hline $\begin{array}{l}\text { 3. Si se me rompiera el celular durante un } \\
\text { período largo de tiempo y tardaran en ar- } \\
\text { reglarlo, me pondría mal. }\end{array}$ & 3.37 & 1.446 & -.463 & -1.165 & .731 & .771 \\
\hline 5. Si no tengo el celular me siento mal. & 2.72 & 1.393 & .142 & -1.277 & .725 & .778 \\
\hline $\begin{array}{l}\text { 10. No creo que pudiera aguantar una se- } \\
\text { mana sin celular. }\end{array}$ & 2.75 & 1.518 & .247 & -1.397 & .690 & .813 \\
\hline \multicolumn{7}{|l|}{ Abuso $(\alpha=.77)$} \\
\hline $\begin{array}{l}\text { 4. Dedico más tiempo del que quisiera a usar } \\
\text { el celular. }\end{array}$ & 3.00 & 1.409 & -.075 & -1.276 & .612 & .690 \\
\hline $\begin{array}{l}\text { 6. Me he acostado más tarde o he dormido } \\
\text { menos por estar utilizando el celular. }\end{array}$ & 2.75 & 1.386 & .089 & -1.299 & .673 & .618 \\
\hline $\begin{array}{l}\text { 9. Utilizo el celular en situaciones que, } \\
\text { aunque no son peligrosas, no es correcto } \\
\text { hacerlo (comiendo, mientras otras personas } \\
\text { me hablan, etc.). }\end{array}$ & 2.67 & 1.249 & .340 & -.886 & .545 & .761 \\
\hline \multicolumn{7}{|l|}{ Tolerancia $(\alpha=.77)$} \\
\hline 2. Últimamente utilizo mucho más el celular. & 3.16 & 1.354 & -.220 & -1.131 & .619 & .683 \\
\hline $\begin{array}{l}\text { 4. Cada vez necesito utilizar el celular con } \\
\text { más frecuencia. }\end{array}$ & 3.00 & 1.409 & -.075 & -1.276 & .670 & .624 \\
\hline $\begin{array}{l}\text { 6. Cuando tengo el celular a mano, no puedo } \\
\text { dejar de utilizarlo. }\end{array}$ & 2.75 & 1.386 & .089 & -1.299 & .540 & .769 \\
\hline \multicolumn{7}{|l|}{ Pérdida de control $(\alpha=.76)$} \\
\hline $\begin{array}{l}\text { 8. Apenas me levanto lo primero que hago } \\
\text { es ver si me han enviado WhatsApps y/o } \\
\text { chequear las redes sociales, etc. }\end{array}$ & 3.60 & 1.471 & -.658 & -1.019 & .543 & .728 \\
\hline $\begin{array}{l}\text { 11. Cuando me siento solo chequeo las redes } \\
\text { sociales, le envío un WhatsApp a alguien, etc. }\end{array}$ & 3.44 & 1.416 & -.497 & -1.064 & .621 & .640 \\
\hline $\begin{array}{l}\text { 12. Ahora mismo agarraría el celular y miraría } \\
\text { las redes sociales o enviaría mensajes. }\end{array}$ & 2.65 & 1.464 & .250 & -1.340 & .603 & .660 \\
\hline
\end{tabular}

Nota. $M=$ Media; $D T=$ Desviación típica; $S=$ Asimetría; $K=$ Curtosis; $r j x=$ Correlación ítem-total; $\alpha .-x=$ Alfa de Cronbach si se elimina el elemento. 
asimetría, la curtosis, y la correlación ítem-total. En cuanto a cada una de las dimensiones, la consistencia interna (alfa de cronbach) resultó adecuada, permitiendo inferir que cada dimensión resultó confiable para su uso en el contexto argentino. Además, en caso de eliminar alguno de los ítems que componen la escala, la confiabilidad de cada una de las dimensiones no mejora significativamente.

Con el objetivo de testear la validez de constructo, se realizó un AFC probando los modelos de una dimensión y el modelo de cuatro dimensiones correlacionadas entre sí, como plantea el autor original de la prueba (Chóliz et al., 2016).

En la Tabla 2, se puede observar un mejor ajuste del modelo de cuatro dimensiones que el modelo de una dimensión. Así, todos los indicadores testeados permiten inferir que para los datos del presente estudio, el modelo multidimensional-cuatro dimensiones-, sería el que presenta un mejor ajuste.

En cuanto al género, se hallaron diferencias estadísticamente significativas en las dimensiones Abstinencia $\left(t_{(337)}=-3,006 ; p<.001 ; d\right.$ de Cohen $\left.=.388\right)$ y Pérdida de Control $\left(t_{(337)}=-2,021 ; p<.001 ; d\right.$ de Cohen $\left.=.248\right)$. En la dimensión Abstinencia, las mujeres $(M=$ 9.22; $D T=3.85)$ obtuvieron mayores puntajes que los hombres $(M=7.79 ; D T=3.50)$. Con respecto a la dimensión Pérdida de Control, las mujeres $(M=9.91 ; D T=3.53)$ también obtuvieron puntajes mayores que los hombres $(M=9.02 ; D T=3.62)$.

Con respecto a la edad de los participantes, se hallaron relaciones significativas negativas con las dimensiones Abuso $(r=-.453 ; p<.001)$, Tolerancia $(r=-.187 ; p<.001)$ y Pérdida de Control $(r=-.329 ; p<.001)$. No se hallaron correlaciones con la dimensión Abstinencia.

Posteriormente, se testearon las relaciones entre las cuatro dimensiones de la TDMB con las cuatro de la Impulsividad (Tabla 3). Los resultados observados en la Tabla 3 indican que la dimensión abstinencia se relacionó significativamente y de manera positiva con la dimensión falta de perseverancia de la escala de impulsividad $(r=.108 ; p<.001)$ y con mayor intensidad con respecto a la dimensión urgencia negativa de la misma escala $(r$ $=.239 ; p<.001)$. Por su parte, la dimensión abuso se relacionó significativamente y de manera positiva con las dimensiones falta de perseverancia de la escala de impulsividad ( $r$ $=.235 ; p<.001)$, urgencia negativa $(r=.355 ; p<.001) \mathrm{y}$, en menor medida, con búsqueda de sensaciones $(r=.128 ; p<.001)$ de la escala de impulsividad. En cuanto a la dimensión tolerancia, se observaron relaciones positivas y significativas con falta de perseverancia $(r$ $=.115 ; p<.001) \mathrm{y}$, en mayor medida, con urgencia negativa de la escala de impulsividad

Tabla 2

Análisis Factorial Confirmatorio de la escala TDMB comparando una y cuatro dimensiones

\begin{tabular}{cccccccc}
\hline & $\mathrm{X}^{2}{ }_{(\mathrm{q})}$ & $\mathrm{S}-\mathrm{B} \mathrm{X}^{2}{ }_{(\mathrm{q})}$ & $\Delta \mathrm{S}-\mathrm{B} \mathrm{X}^{2}{ }_{(\mathrm{ql})}$ & $\mathrm{NNFI}$ & $\mathrm{CFI}$ & IFI & RMSEA \\
\hline $\begin{array}{c}\text { TDMB (una } \\
\text { dimensión) }\end{array}$ & $458.196_{(54)}$ & $381.109_{(54)}$ & 7.05 & .80 & .83 & .83 & .134 \\
& & & & & & & {$[.121-.146]$} \\
$\begin{array}{c}\text { TDMB (cuatro } \\
\text { dimensiones } \\
\text { correlacionadas) }\end{array}$ & $154.043_{(48)}$ & $136.043_{(48)}$ & 2.83 & .94 & .95 & .95 & .074 \\
\hline
\end{tabular}

Nota. TDMB (una dimensión): Modelo unidimensional; TDMB (cuatro dimensiones correlacionadas): Modelo de cuatro dimensiones correlacionadas. Valores adecuados: $\Delta S-B \chi_{(g l)}^{2} \leq 5 ; \mathrm{NNFI}$, $C F I, I F I \geq .90 ;$ RMSEA $\leq .08$. 


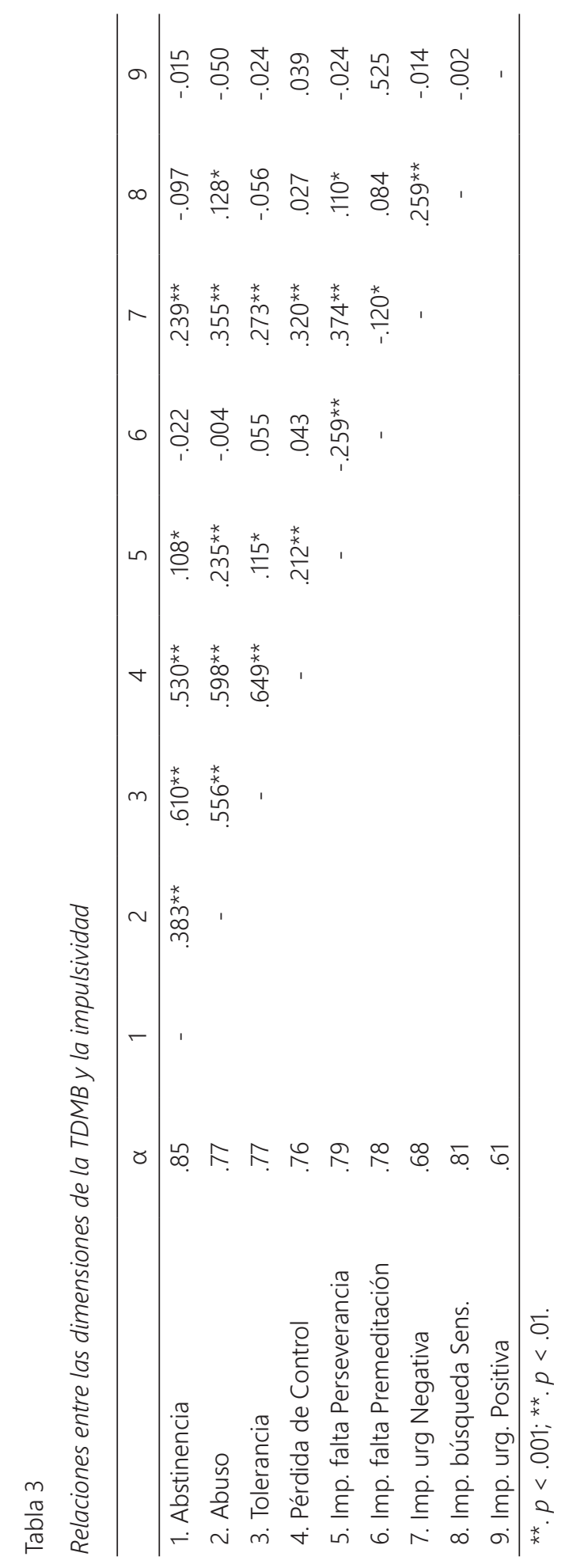

Actualidades en Psicología, 35(130), 2021, 1-18 


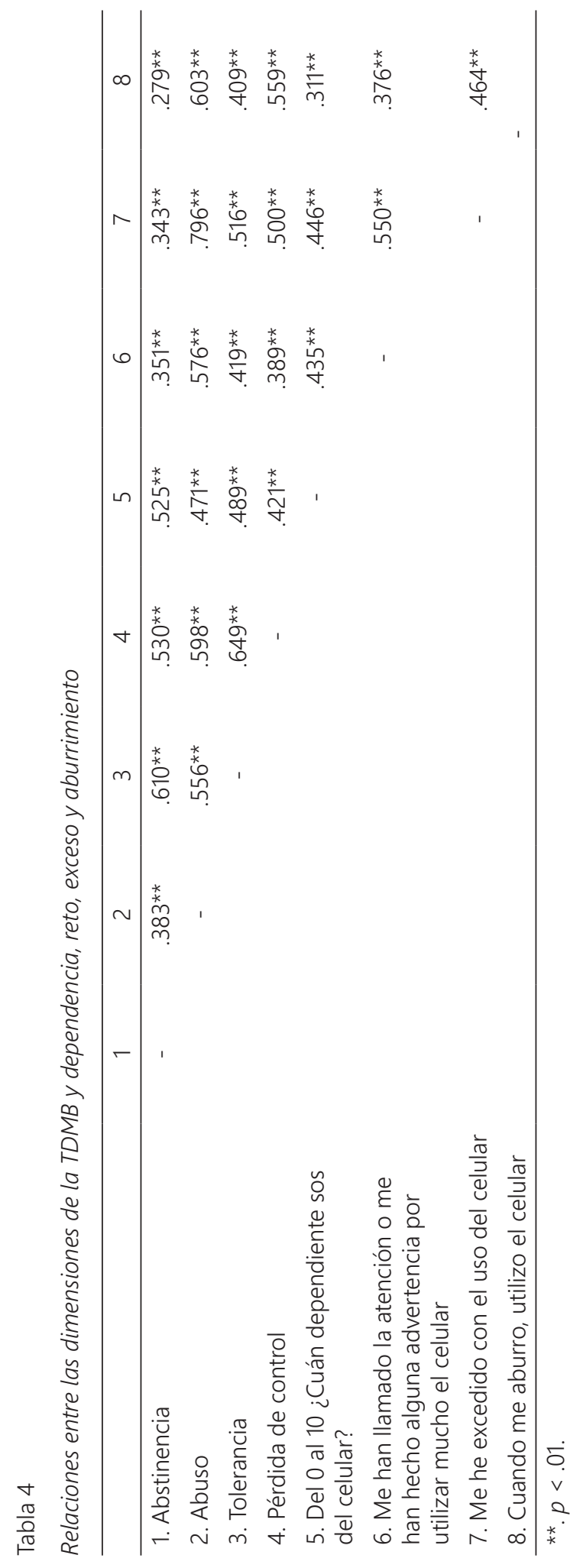

Actualidades en Psicología, 35(130), 2021, 1-18 
$(r=.273 ; p<.001)$. Por último, la dimensión pérdida de control se relacionó positiva y significativamente con las dimensiones impulsividad y falta de perseverancia de la escala breve de comportamiento impulsivo UPPS-P $(r=.212 ; p<.001) \mathrm{y}$, en mayor medida, con urgencia negación de la UPPS-P $(r=.320 ; p<.001)$.

Finalmente, se observaron las relaciones entre las dimensiones de la TDMB y cuatro indicadores sobre el uso del teléfono móvil como la autopercepción de dependencia, si terceros notaron un uso excesivo, la autopercepción de excederse y el uso del móvil como entretenimiento (Tabla 4).

Las correlaciones fueron positivas y significativas entre las cuatro dimensiones y cada uno de los indicadores evaluados. Fueron mayores aquellas entre la dimensión abstinencia y la autopercepción de dependencia $(r=.525 ; p<.001)$, así como entre la dimensión abuso con los llamados de atención o advertencia ( $r=.576 ; p<.001)$, la autopercepción de exceso en el uso del celular $(r=.796 ; p<.001)$ y el uso en situaciones de aburrimiento $(r=.603 ; p<.001)$.

\section{Discusión}

El objetivo principal de este trabajo pudo cumplirse, dado que los resultados observados corroboran que el instrumento TDM Brief, al igual que lo evidenciado en estudios previos (Chóliz, 2010; Chóliz et al., 2016), es válido y confiable para su uso en el contexto argentino. Además, las propiedades psicométricas observadas son consistentes con las halladas en otras adaptaciones de la prueba, por ejemplo, en cuanto a la solución de cuatro factores correlacionados, la cual también fue hallada en la creación de la prueba (Chóliz et al., 2016), como en la adaptación al contexto peruano (Gamero et al., 2016) y brasilero (Flores et al., 2013).

En cuanto al sexo, en este estudio se hallaron diferencias estadísticamente significativas en las dimensiones Abstinencia y Pérdida de Control. En ambas, las mujeres obtuvieron puntajes significativamente mayores que los hombres. Estos resultados son consistentes con los obtenidos por Chóliz et al. (2016), permitiendo arrojar mayor validez a la adaptación del cuestionario realizada en el presente estudio. Tanto en este estudio como en otros desarrollados previamente (Villafuerte-Garzón \& Vera-Perea, 2019), se pudo observar que, si bien hombres y mujeres evalúan positivamente el uso de dispositivos tecnológicos con la finalidad de estar conectados con su familia, las mujeres han tenido mayor frecuencia de uso que los hombres.

Estos hallazgos también son consistentes con aquellos identificados por Ruiz-Palmero et al. (2016), quienes observaron diferencias significativas en los factores Uso desmedido y Abstinencia, donde las mujeres tienen más dependencia que los hombres a los teléfonos móviles. Estos hallazgos también se han visto reflejados en el estudio de De la Villa Moral y Suárez (2016), quienes confirmaron la existencia de diferencias estadísticamente significativas en el factor que mide problemas relacionados con el uso emocional y comunicativo del teléfono móvil, donde nuevamente las mujeres presentaron un mayor uso problemático.

Con respecto a la edad de los participantes, se hallaron relaciones negativas y significativas con las dimensiones abuso, tolerancia y pérdida de control, pero no con la dimensión abstinencia (es decir, a menor edad, mayor abuso, tolerancia y pérdida de control). Esto podría indicar que, para la muestra con la que se trabajó, la abstinencia no es un factor que esté asociado a la edad de los individuos. No obstante, la edad se 
correlacionó positivamente con la percepción de problemas, tendencia que también ha sido confirmada en el estudio de Labrador y Villadangos (2010), quienes interpretaron que ante una mayor frecuencia de uso se perciben o se han experimentado más problemas, o bien que, conforme aumenta la edad, se van identificando mejor los efectos negativos.

Luego de testear las relaciones entre las cuatro dimensiones de la TDMB -abstinencia, abuso e interferencia con otras actividades, tolerancia y falta de control- con los cinco rasgos relacionados con el comportamiento impulsivo UPPS-P, a saber: urgencia positiva, urgencia negativa, falta de premeditación, falta de perseverancia y búsqueda de sensaciones, se observó que la dimensión abstinencia de la TDMB se relaciona significativamente y de manera positiva con la dimensión falta de perseverancia de la UPPS-P, y lo hizo con mayor intensidad que con la dimensión urgencia negativa de la misma escala. Esto podría indicar que un importante factor de riesgo en el uso excesivo de celular es la impulsividad como rasgo, así como una alta sensibilidad al refuerzo inmediato con incapacidad de aplazamiento (Hogarth, 2011) y la necesidad de búsqueda de sensaciones. Además, la impulsividad se relaciona con conductas adictivas como el uso excesivo del celular, excesiva conexión a Internet y redes sociales, abuso de alcohol, conductas sexuales y el gasto compulsivo (Billieaux et al., 2010).

Por su parte, la dimensión abuso de la TDMB se relaciona significativamente y de manera positiva con las dimensiones falta de perseverancia y urgencia negativa de la UPPS-P y, en menor medida, con búsqueda de sensaciones de la misma escala. En las personas que experimentan dificultad en el control de los impulsos, con estados de tensión y con el objetivo final de conseguir un refuerzo gratificante que se plasma en el consumo en el uso de celular o la conexión excesiva a redes sociales (De Sola Gutiérrez et al., 2013), la falta de control de impulsos marca una tendencia a realizar la acción sin tomar conciencia de las consecuencias negativas de los actos (Moeller et al., 2001). La impulsividad sería un acto irresistible en el que se experimenta un deseo intenso de realizar acciones que el sujeto sabe que son desencadenantes de consecuencias negativas (Salvo \& Castro 2013).

Estos comportamientos responderían al modelo del circuito del refuerzo de Koob y Volkow (2010), en donde el estado de emergencia derivado de la disforia o lado afectivo negativo se situaría justo en el tránsito de la impulsividad a la compulsión y vendría marcado por el cambio del refuerzo positivo al negativo. Es decir, la impulsividad precederá y se encontraría en la base de un comportamiento impulsivo que llevaría a falta de control de la conducta (Verdejo-García et al., 2008; Belin et al., 2011; Papachristou et al., 2012; Everitt et al., 2008; Hogarth, 2011; Michalczuk et al., 2011). Desde este planteamiento, teniendo en cuenta que el circuito del refuerzo se encuentra activado constantemente, potencialmente en personas con vulnerabilidad en el control de los impulsos, cualquier conducta podría llegar a convertirse en una adicción (Beck et al., 2010). La cuestión, sin embargo, es el daño o interferencia que sería capaz de producir.

En cuanto a las dimensiones tolerancia y pérdida de control de la TDMB, se han observado también relaciones positivas y significativas con falta de perseverancia y, en mayor medida, con urgencia negativa de la escala de impulsividad. Lo anterior evidencia que, para los participantes del estudio, la abstinencia, tolerancia, dependencia y dificultad de control podrían relacionarse con la necesidad de disminuir estados de ánimo disfóricos (Hooper \& Zhou, 2007; Leung, 2008; Jones, 2014). Cuando no es posible el acceso continuo al teléfono móvil, se generan sensación de urgencia y necesidad de constantes chequeos al celular, con estrés, ansiedad y cambios de humor (Fondevila et al., 2014; Ha et al., 2008; Tosell et al., 2015), así como insomnio y trastornos del sueño (Sahin et al., 2013). 
Por último, tras analizar las relaciones entre las dimensiones de la TDMB y cuatro indicadores en relación con el uso de celular, se observó que estas fueron positivas y significativas. En esta línea, cabe destacar lo que distintos autores han señalado como otras consecuencias negativas derivadas del uso del teléfono celular, tales como inquietud, irritabilidad o sentimientos de estar perdido si no se dispone del aparato (Chóliz, 2010, 2012; Chóliz et al., 2009; Kwon et al., 2013); abuso y uso consciente en situaciones peligrosas o contextos prohibidos (Billieux et al., 2008; Sánchez-Carbonell et al., 2008; Backer-Grondahl \& Sagberg, 2011); conflictos y daños físicos, mentales, sociales, laborales o familiares (Chesley, 2005; Chóliz, 2012) con pérdida de interés en otras actividades aun a pesar de los efectos negativos que esto conlleva (Labrador \& Villadangos, 2010).

Como se indicó al principio de este trabajo, si bien la impulsividad en el DSM-V se encuentra en la categoría de trastornos disruptivos, del control de los impulsos y otras alteraciones (Botero-Franco et al., 2016), se presenta una modificación que reemplaza el término 'adicción' por los 'Trastornos de uso y abuso' y añade el 'craving', o deseo intenso de consumo, como otro nuevo criterio de estos trastornos. No obstante, todavía no existe un consenso universalmente validado de 'craving' en los trastornos por uso excesivo de tecnología, ya que es un concepto que ha surgido de las adicciones a sustancias.

Hay nueva terminología que no figura en los manuales diagnósticos para dar cuenta de los fenómenos relacionados al abuso o uso excesivo del celular, como Fear of Missing Out o expresión del temor a encontrarse sin el celular, desconectado de sus amigos y salidas o fuera de cobertura; Nomofobia (No-Mobile-Phobia), Ringxiety y Textafrenia, que hacen referencia a la falsa sensación de haber recibido un mensaje de texto o una llamada que lleva al chequeo constante del celular, así como Textiety o ansiedad de recibir y responder inmediatamente a los mensajes de texto (Taneja, 2014). Sin embargo, resulta difícil determinar si Internet y las Nuevas Tecnologías en general son capaces de suscitar comportamientos adictivos o son, por el contrario, generados por los contenidos. Es una posibilidad que Internet pueda ser un canal y vehículo de adicciones específicas tales como el juego, las compras o el sexo y no el medio tecnológico per se (Ma, 2011).

A través de esta herramienta de evaluación, los profesionales de la salud mental podrán trazar una línea más clara entre el uso de teléfonos celulares adaptativos y desadaptativos, así como diseñar planes de tratamiento más eficientes y, a su vez, permitiría comprender mejor la complejidad de esta adicción comportamental en todas sus dimensiones.

\section{Referencias}

Anestis, M. D., Selby, E. A., Fink, E. L., \& Joiner, T. E. (2007). The multifaceted role of distress tolerance in dysregulated eating behaviors. International Journal of Eating Disorders, 40(8), 718-726. https://doi.org/10.1002/eat.20471

Asociación Americana de Psiquiatría (2018). Manual Diagnóstico y Estadístico de Trastornos mentales (DSM-5®). American Psychiatric Pub.

Backer-Grøndahl, A., \& Sagberg, F. (2011). Driving and telephoning: Relative accident risk when using hand-held and hands-free mobile phones. Safety Science, 49(2), 324330. https://doi.org/10.1016/j.ssci.2010.09.009

Beck, A. T., Rush, A. J., Shaw, B. F., \& Emery, G. (2010). Terapia cognitiva de la depresión. Desclée de Brouwer.

Actualidades en Psicología, 35(130), 2021, 1-18 
Becoña, E. (2016). La adicción "no" es una enfermedad cerebral. Papeles del psicólogo, 37(2), 118-125.

Belin, D., Berson, N., Balado, E., Piazza, P. V., \& Deroche-Gamonet, V. (2011). High-noveltypreference rats are predisposed to compulsive cocaine self-administration. Neuropsychopharmacology, 36(3), 569. https://doi.org/10.1038/npp.2010.188

Bianchi, A., \& Phillips, J. G. (2005). Psychological predictors of problem mobile phone use. CyberPsychology \& Behavior, 8(1), 39-51. https://doi.org/10.1089/cpb.2005.8.39

Billieux, J., Van der Linden, M., \& Rochat, L. (2008). The role of impulsivity in actual and problematic use of the mobile phone. Applied Cognitive Psychology, 22(9), 11951210. https://doi.org/10.1002/acp.1429

Billieux, J., Gay, P., Rochat, L., \& Van der Linden, M. (2010). The role of urgency and its underlying psychological mechanisms in problematic behaviours. Behaviour research and therapy, 48(11), 1085-1096. https://doi.org/10.1016/j. brat.2010.07.008

Billieux, J. (2012). Problematic use of the mobile phone: a literature review and a pathways model. Current Psychiatry Reviews, 8(4), 299-307. https://doi. org/10.2174/157340012803520522

Botero-Franco, D., Palacio-Ortíz, J. D., Arroyave-Sierra, P., \& Piñeros-Ortíz, S. (2016). Clinical Implications of Changes in Child Psychiatry in the DSM-5. Strengths and Weaknesses of the Changes. Revista colombiana de psiquiatría, 45(3), 201-213. https://doi.org/10.1016/j.rcp.2015.08.001

Cándido, A., Orduña, E., Perales, J. C., Verdejo-García, A. \& Billieux, J. (2012). Validation of a short Spanish version of the UPPS-P impulsive behaviour scale. Trastornos Adictivos, 14, 73-78.

Carbonell, X. (2014). La adicción a los videojuegos en el DSM-5. Adicciones, 26(2), 91-95. https://doi.org/10.20882/adicciones.10

Carbonell, X., \& Oberst, U. (2015). Las redes sociales en línea no son adictivas. Aloma, 33(2), 13-19.

Caro, M. (2017). Adicciones tecnológicas: ¿Enfermedad o conducta adaptativa? Revista Medisur, 15(2), 251-260.

Cía, A. (2013). Las adicciones no relacionadas a sustancias (DSM-5, APA, 2013): un primer paso hacia la inclusión de las adicciones conductuales en las clasificaciones categoriales vigentes. Revista de Neuro-Psiquiatria 76(4), 210-217. https://doi. org/10.20453/rnp.2013.1169

Chesley, N. (2005). Blurring boundaries? Linking technology use, spillover, individual distress, and family satisfaction. Journal of Marriage and Family, 67(5), 1237-1248. https://doi.org/10.1111/j.1741-3737.2005.00213.x

Chóliz, M., Villanueva, V., \& Chóliz, M. C. (2009). Ellas, ellos y su móvil: Uso, abuso (¿y dependencia?) del teléfono móvil en la adolescencia. Revista española de drogodependencias, 34(1), 74-88.

Chóliz, M. (2010). Mobile phone addiction: a point of issue. Addiction, 105(2), 373-374. https://doi.org/10.1111/j.1360-0443.2009.02854.x 
Chóliz, M. (2012). Mobile-phone addiction in adolescence: the test of mobile phone dependence (TMD). Progress in Health Sciences, 2(1), 33-44.

Chóliz, M., Pinto, L., Phansalkar, S. S., Corr, E., Mujjahid, A., Flores, C., \& Barrientos, P. E. (2016). Development of a brief multicultural version of the test of mobile phone dependence (TMD brief) questionnaire. Frontiers in psychology, 7, 650. https:// doi.org/10.3389/fpsyg.2016.00650

Chóliz, M., Echeburúa, E., \& Ferre, F. (2017). Screening Tools for Technological Addictions: A Proposal for the Strategy of Mental Health. International Journal of Mental Health and Addiction, 15(2), 423-433. https://doi.org/10.1007/s11469-017-9743-1

De la Villa Moral, M., \& Suárez, C. (2016). Factores de riesgo en el uso problemático de Internet y del teléfono móvil en adolescentes españoles. Revista Iberoamericana de Psicología y Salud, 7(2), 69-78. https://doi.org/10.1016/j.rips.2016.03.001

De Sola Gutiérrez, J., Valladolid, G. R., \& de Fonseca, F. R. (2013). La impulsividad: ¿Antesala de las adicciones comportamentales? Salud y drogas, 13(2), 145-155. https://doi. org/10.21134/haaj.v13i2.211

Echeburúa, E. (1999). Cambio de concepto. Revista Española, 24(1), 329-331.

Everitt, B. J., Belin, D., Economidou, D., Pelloux, Y., Dalley, J. W., \& Robbins, T. W. (2008). Neural mechanisms underlying the vulnerability to develop compulsive drugseeking habits and addiction. Philosophical Transactions of the Royal Society. Biological Sciences, 363(1507), 3125-3135. https://doi.org/10.1098/rstb.2008.0089

Flores, N., Jenaro, C., González, F., Martín, E., \& Poy, R. (2013). Adicción al móvil en alumnos de secundaria: efectos en la convivencia. European Journal of Investigation in Health, Psychology and Education, 3(3), 215-225.

Fondevila, J. F., Carreras, M., Mir, P., del Olmo, J. L., \& Pesqueira, M. J. (2014). El impacto de la mensajería instantánea en los estudiantes en forma de estrés y ansiedad para el aprendizaje: análisis empírico. Didáctica, innovación y multimedia, (30), 1-15.

Gamero, K., Flores, C., Arias, W. L., Ceballos, K. D., Román, A., \& Marquina, E. (2016). Estandarización del Test de Dependencia al Celular para estudiantes universitarios de Arequipa. Persona, (019), 179-200. https://doi.org/10.26439/persona2016. n019.979

Guertler, D., Broda, A., Bischof, A., Kastirke, N., Meerkerk, G.-J., John, U., \& Rumpf, H.-J. (2014). Factor Structure of the Compulsive Internet Use Scale. Cyberpsychology, Behavior, and Social Networking, 17(1), 46-51. https://doi.org/10.1089/cyber.2013.0076

Grant, J. E., Potenza, M. N., Weinstein, A., \& Gorelick, D. A. (2010). Introduction to behavioral addictions. The American journal of drug and alcohol abuse, 36(5), 233-241. https://doi.org/10.3109/00952990.2010.491884

Griffiths, M. (2000). Internet addiction-time to be taken seriously? Addiction research, 8(5), 413-418. https://doi.org/10.3109/16066350009005587

Griffiths, M., Kuss, D., \& King, D. (2012). Video game addiction: Past, present and future. Current Psychiatry Reviews, 8(4), 308-318.

Gutiérrez, F. G. (2014). La "revolución" de las redes sociales: sociedad, educación y nueva profesión. Voces en el fénix, (40), 112-119. 
Ha, J. H., Chin, B., Park, D. H., Ryu, S. H., \& Yu, J. (2008). Characteristics of excessive cellular phone use in Korean adolescents. Cyber Psychology \& Behavior, 11(6), 783-784. https://doi.org/10.1089/cpb.2008.0096

Hambleton, R. K. (1994). Guidelines for adapting educational and psychological tests: A progress report. European Journal of Psychological Assessment, 10(3), 229-244.

Hogarth, R. M. (2011). On the learning of intuition. In H. Plessner, C. Betsch, \& T. Betsch (Eds.), Intuition in judgment and decision making (pp. 111-126). Psychology Press.

Hooper, V., \& Zhou, Y. (2007). Addictive, dependent, compulsive? A study of mobile phone usage [Conference presentation]. 20th Bled eConference eMergence: Merging and Emerging Technologies, Processes, and Institutions, Bled, Slovenia.

Igarashi, T., Motoyoshi, T., Takai, J., \& Yoshida, T. (2008). No mobile, no life: Self-perception and text-message dependency among Japanese high school students. Computers in Human Behavior, 24(5), 2311-2324. https://doi.org/10.1016/j.chb.2007.12.001

Instituto Nacional de Estadísticas y Censos (2017). Censo Nacional de Población, Hogares y Viviendas. INDEC.

Jones, T. (2014). Students' cell phone addiction and their opinions. The Elon Journal of Undergraduate Research in Communications, 5(1), 74-80.

Koob, G. F., \& Volkow, N. D. (2010). Neurocircuitry of addiction. Neuropsychopharmacology, 35(1), 217-238. https://doi.org/10.1038/npp.2009.110

Kwon, M., Lee, J. Y., Won, W. Y., Park, J. W., Min, J. A., Hahn, C., \& Kim, D. J. (2013). Development and validation of a smartphone addiction scale (SAS). PloS one, 8(2), e56936. https://doi.org/10.1371/journal.pone.0056936

Labrador, F. E., \& Villadangos, S. G. (2010). Adolescents and new technologies: Behaviours pointing a possible addiction problem. Psicothema, 22(2), 180-188.

Leung, L. (2008). Linking psychological attributes to addiction and improper use of the mobile phone among adolescents in Hong Kong. Journal of children and media, 2(2), 93-113. https://doi.org/10.1080/17482790802078565

Ma, H. K. (2011). Internet addiction and antisocial internet behavior of adolescents. The Scientific World Journal, 11. https://doi.org/10.1100/2011/308631

Matute, H. (2016). ¿Adicción, abuso o uso problemático de Internet? En Echeburúa, E. (Coord.). Abuso de internet: ¿antesala para la adicción al juego de azar online? (pp. 31-50). Pirámide.

Michalczuk, R., Bowden-Jones, H., Verdejo-Garcia, A., \& Clark, L. (2011). Impulsivity and cognitive distortions in pathological gamblers attending the UK National Problem Gambling Clinic: a preliminary report. Psychological medicine, 41(12), 2625-2635. https://doi.org/10.1017/S003329171100095X

Miller, G. (2012). The smartphone psychology manifesto. Perspectives on psychological science, 7(3), 221-237. https://doi.org/10.1177/1745691612441215

Moeller, F. G., Barratt, E. S., Dougherty, D. M., Schmitz, J. M., \& Swann, A. C. (2001). Psychiatric aspects of impulsivity. American journal of psychiatry, 158(11), 17831793. https://doi.org/10.1176/appi.ajp.158.11.1783 
Muñiz, J., Elosua, P., \& Hambleton, R. K. (2013). International Test Commission Guidelines for test translation and adaptation. Psicothema, 25(2), 151-157. https://doi. org/10.7334/psicothema2013.24

Organización Mundial de la Salud (OMS). 2018. Clasificación Internacional de Enfermedades, 11.a revisión.

Papachristou H., Nederkoorn C., Havermans R., Van Der Horst M., \& Hansen A. (2012). Can't stop the craving: The effect of impulsivity on cue-elicited craving for alcohol in heavy and light social drinkers. Psychopharmacology, 219, 511-518. https://doi. org/10.1007/s00213-011-2240-5

Pedrero-Pérez, E., Ruíz-Sánchez de León, J. M., Rojo-Mota, G., Llanero-Luque, M., Pedrero-Aguilar, J., Morales-Alonso, S., \& Puerta-García, C. (2018). Tecnologías de la Información y la Comunicación (TIC): abuso de Internet, videojuegos, teléfonos móviles, mensajería instantánea y redes sociales mediante el MULTICAGE-TIC. Adicciones. Revista online, 30(1), 19-32. https://doi.org/10.20882/adicciones.806

Ruiz-Palmero, J., Sánchez-Rodríguez, J., \& Trujillo-Torres, J. M. (2016). Utilización de Internet y dependencia a teléfonos móviles en adolescentes. Revista Latinoamericana de Ciencias Sociales, Niñez y Juventud, 14(2), 1357-1369.

Sahin, S., Ozdemir, K., Unsal, A., \& Temiz, N. (2013). Evaluation of mobile phone addiction level and sleep quality in university students. Pakistan journal of medical sciences, 29(4), 913-918. https://doi.org/10.12669/pjms.294.3686

Salvo, L., \& Castro, A. (2013). Soledad, impulsividad, consumo de alcohol y su relación con suicidalidad en adolescentes. Revista médica de Chile, 141(4), 428-434. https:// doi.org/10.4067/S0034-98872013000400002

Sánchez-Carbonell, X., Beranuy, M., Castellana, M., Chamarro, A., \& Oberst, U. (2008). La adicción a Internet y al móvil: ¿moda o trastorno? Adicciones, 20(2), 149-159. https://doi.org/10.20882/adicciones.279

Simó, C., Martínez, A., Ballestar, M. L., \& Domínguez, A. (2017). Instrumentos de evaluación del uso problemático del teléfono móvil/smartphone. Salud y Drogas, 17(1), 5-14.

Starcevic, V. (2012). Is Internet addiction a useful concept? Australian \& New Zeland Journal of Psychiatry 47(1), 16-19. https://doi.org/10.1177/0004867412461693

Taneja, C. (2014). The psychology of excessive cellular phone use. Delhi Psychiatry, 17(2), 448-451.

Toda, M., Monden, K., Kubo, K., \& Morimoto, K. (2006). Mobile phone dependence and health-related lifestyle of university students. Social Behavior and Personality, 34(10), 1277-1284. https://doi.org/10.2224/sbp.2006.34.10.1277

Tosell, C., Kortum, P., Shepard, C., Rahmati, A., \& Zhong, L. (2015). Exploring smartphone addiction: insights from long-term telemetric behavioral measures. International Journal of Interactive Mobile Technologies, 9(2), 37-43. https://doi.org/10.3991/ ijim.v9i2.4300

Tsai, C. C., \& Lin, S. S. (2003). Internet addiction of adolescents in Taiwan: an interview study. Cyberpsychology Behaviour, 6(6), 649-652. https://doi. org/10.1089/109493103322725432

Actualidades en Psicología, 35(130), 2021, 1-18 
Turel, O., \& Serenko, A. (2012). The benefits and dangers of enjoyment with social networking websites. European Journal of Information Systems, 21(5), 512-528. https://doi.org/10.1057/ejis.2012.1

Valencia-Ortiz, R., \& Cabero-Almenara, J. (2019). Adicción a las redes sociales: Validación de un instrumento en el contexto mexicano. Health and Addictions/Salud $y$ Drogas, 19(2), 149-159. https://doi.org/10.21134/haaj.v19i2.460

Verdejo-García, A. J., Perales, J. C., \& Pérez-García, M. (2007). Cognitive impulsivity in cocaine and heroin polysubstance abusers. Addictive behaviors, 32(5), 950-966. https://doi.org/10.1016/j.addbeh.2006.06.032

Verdejo-García, A., Lawrence, A. J., \& Clark, L. (2008). Impulsivity as a vulnerability marker for substance-use disorders: review of findings from high-risk research, problem gamblers and genetic association studies. Neuroscience \& Biobehavioral Reviews, 32(4), 777-810. https://doi.org/10.1016/j.neubiorev.2007.11.003

Villafuerte-Garzón, C. M., \& Vera-Perea, M. (2019). Phubbing y género en un sector académico en Quito: Uso, abuso e interferencia de la tecnología. Convergencia, 26(79). 